\title{
The Effects of Water-Based Exercises on Physiological Status in Degenerative Disease- Patients
}

\author{
MostafaMohamadNour *, Hussein A. Abdel-Salam**
}

This study aims to determine water-based exercise efficiency in improving physiological status in degenerative disease patinets. The sample of 20 subjects (age: $42 \pm 3$ yr, height: $172 \pm 4 \mathrm{~cm}$, weight: $95 \pm 9$ $\mathrm{kg}$ ) has been randomly assigned to control and experimental group. The experimental group was subjected to six week training program, consisted of aerobic water-based exercises (35-70\% of heart rate reserve). The control group was only habitualy active during this period. Before and after training program, blood glucose, cholesterol, triglyceride, body weight and resting, maximum and recovery heart rates responses in multi-stage treadmill test were measured in the two groups. There were significant improvements in the measured physiological research variables in the experimental group, compared to the control group. Blood glucose, cholesterol and triglyceride had decreased in the experimental group by $24 \%, 13 \%$ and $27 \%$, respectively. In conclusion, it can be stated that 6 week of aerobic water-based exercise program significantly improves the physiological functions in degenerative disease-patients.

\section{Introduction}

A ccording to $\mathrm{WHO}$, degenerative disease is $A$ a failure in the human body being caused by environmental conditions or genetics. Exercises specialists have long been interested how to cure or prevent such conditions. The results of their collective work were translated into a multitude of exercise programs.

Although these programs were carried out in different environments, applying various techniques and methods, there is evident lack of findings considering effects of water-based exercise programs on degenerative diseases. The use of the aqueous medium is therefore the focus of the present study which targets individuals attacked by some degenerative diseases. While protecting against such diseases and reducing their severity are the focus of the study, preventing their complications is no less important.

The American Heart Association suggested that

*Assistant Professor, Exercises and Gymnastics Training Department,The Faculty of Physical Education, Abu Qir, Alexandria University, Egypt.

**Assistant Professor, Aquatic Sports Training Department, The Faculty of Physical Education, Abu Qir, Alexandria University, Egypt. the organized physical training, especially lowintensity physical exercise, increases the fitness of various vital organs and, in turn, plays a major role in preventing degenerative diseases. Recently, there has been an increasing awareness of the value of using the aqueous medium on such conditions.

\section{Benefits of water-based exercises}

The aqueous medium is a good means of fitness, rehabilitation, physical therapy and preventive medicine. Physical exercises that use this medium, have the following benefits:

- Increasing the ability to work, as the aqueous exercises are refreshing and help improve an individual's performance; Comment: rephrase, not clear?

- Reducing the chances for joints and muscles injuries. A number of studies proved that exercises done in an aqueous medium are more suitable than exercises done on the ground for certain people because the weight-bearing pressure on the lower limb is much lower in aqueous medium than on the ground;

- Improving performance as the exercises in the aqueous medium enables a person to do 
the exercises with lightness and agility; Comment: rephrase, not clear?

- The aqueous medium exercises are applied as if they were a rule for all new theories and scientific valuable techniques of the training method. Comment- exclude, not relevant

- The possibility of doing the exercises in any aquatic environment and suitability for all age groups from childhood to old age; explain?

- Providing a physiological, psychological and entertaining help;

- Making joints more flexible and making muscles stronger while minimizing the risk of injury through exercises that work against water resistance by using gradual weights;

- The important role played by such exercises in developing health physiologically in general and keeping the vital organs of the body working in particular; rephrase, not clear

Bearing in mind that medical treatment aims at recovering deteriorated vital organs or addressing system failures, the authors of the present study, working in the field of physical education and sport science, have designed a program that can be applied in an aqueous medium and that depend on the human body's movement, with the aim to improve the functioning of all the vital organs of the body. without threatening the body mechanisms.

Designed for persons suffering from some degenerative diseases, the program reflects special specifications in conformity with the medical science and the biological and mechanical facts. It also has an emotional component related to psychological stability, mental activity, intellectual production and self confidence. This all contributes to a feeling of being able to make achievements, perform daily tasks and increases productivity.

\section{Research Terminology Cardiovascular Diseases}

Cardiovascular diseases have emerged in the modern times as the leading cause of death and are nowon top of the all the degenerative diseases, including the following:

\section{Hypertension:}

According to Eugene $(5: 121)$, it was notedthat hypertensionrate is higher in crowded, bustling citiesthaninsmaller townsandrural areas. It was also notedthat someraces are more prone to hypertensionthan others.

\section{Angiosclerosis:}

Douglas $(8: 78)$ says that this disease is the result ofphysiological changesinblood vessels thatresult inplaque deposits build up ofcholesterol fat films on artery inner-walls rightunder the overlay. This in turn causes a hardening of the artery that helpsblood to clotgraduallyand causea clotblockingthe artery. As the arteries walls thicken, the pathway for blood flow tends to narrow gradually. This reduces or completely block the blood flow and hinders effective blood circulation depriving the body organs of oxygenated blood.

\section{Angina Pectoris:}

This disease, according to Smith Wynn, is caused by narrowing of the coronary artery, which supplies the body organs with oxygenated blood, due to increased plaque deposits build up of cholesterol fat films. Eventually, less blood reaches the heart.

\section{Thrombosis:}

Stein and Mary state $(22: 185)$ that thrombosis is due to the formation of blood clots inside an artery obstructing the flow of blood through the arteries. If the levels of coagulation is elevated this will lead to blocking of arteries. In case this happens directly in the coronary artery, it will cause an unexpected mortality. Thrombosis happens in the leg veins. It doesn't cause symptoms at the beginning. If the clotting is too severe and the clot breaks free, the clot is now known as an embolus. The embolus travels upwards to the thigh and continues its traveling towards the heart and increases in volume and it becomes more severe. If this disturbed blood comes to the heart, it blocks the coronary artery and causes death.

Diabetes: 
Diabetes is a chronic disease that occurs either when the pancreas does not produce enough insulin or when the body cannot effectively use the insulin it produces. The WHO (23:13) stated that diabetes is most wide-spread disease worldwide and especially in the developing countries. Diabetes is considered the most serious disease nowdays.

Although the $30-50$ year age span is the most energetic and fruitful period in man's life, unfortunately it is during this very period that health is compromised due to inefficient body organs caused by the aging process and hindered metabolic rate, activity ofendocrineglands, and the nervous system..

\section{Objectives of the study:}

This research aims to Identify the effects of aerobic Water-based exercises in improving blood glucose, cholesterol and triglyceride levels, heart rate responses before, during and after 2, 4 and 6 minutes of multi-stage treadmill test; time of effort resistance per minute and body weight in degenerative diseased patients.

\section{Hypotheses:}

The authors assume that middle-aged individuals performing the proposed training program on a regular basis improve their physical and psychological capacity represented in the following variables: diabetes; cholesterol; triglyceride; heart beat rate during rest and effort; time of effort resistance per minute; recovery heart rate after 2, 4 and 6 minutes; body weight.

\section{Procedures:}

Methodology: the two-group experimental method: an experimental group and a control group.

\section{Research Scopes:}

Geographical scope: Sport Center in Al-Jouf region in Saudi Arabia.

Human scope: Al-Jouf University associates and some people of the Jouf region in Saudi Arabia.

Time scope: 15.5 .2011 - 30.9.2011

\section{Research Sample:}

The study was conducted on a sample of 20 subjects ( uears, height, weight?) with some degenerative deseases. the sample subjects were randomly divided into control and experimental group $(\mathrm{N}=10)$. This sample was chosen for the following reasons:

- availability of a sporting center;

- having approximately the same social and cultural levels;

- willingness to take the proposed training program regularly

Research Tests: Comment: in order to improve study reproducibility, test protocols should be thoroughly presented, in details!!! Please do so!

A multi-stage physical effort test was performed on the treadmill in order to measure the following variables: heart beat rate during rest and effort; time of effort resistance per minute; recovery heart rate after 2, 4 and 6 minutes.

Measuring Equipment and Tools:

- Treadmill

- Medical balance to take weights

- Restameter to measure height

- Plastic loads (of different weights)

Criteria of selecting of the program exercises:

- Exercises must be of a kinetic type. ???

- Excluding exercises that require holding back one's breath

- Exercises are performed within the limits of the physical achievement capacity of the individual during his own physical effort test.

- Exercises must involve all muscle groups of the body.

- The training must be done individually in a group with no competition between participants. How this is controled???

- Regularity and persistence in training.

- Exercises must be preceded by the basic warm-up exercises to energize the 
circulation of the blood and prepare joints for work

\section{The Study design}

\section{Pre - measurements:}

Pre - measurements were taken of the experimental and control groups of the sample, and relevant data was collected individually for each participant.

\section{Program application:}

The experimental group applied the exercise program in the low-intensity interval training method for a period of six weeks, at the rate of three times per week. The program consisted of the following :

\section{Warm-up :}

(Standing) walking forward around the swimming pool

(Standing) jogging around the swimming pool

(Standing) arms are raised in front (the water on waist level)

(Standing. Arms aside) moving the arms forwards

(Standing. (with hands on waist) push your knees upwards alternately

(Standing. hands thrown forward while touching the swimming pool edge) push your legs up to the sides alternately

(Standing. The back facing the swimming pool edge) push your legs up and forwards alternately

(Standing with hands on waist) bend your trunk to the sides alternately

(Standing with hands on waist) bend your trunk backwards

\section{The main exercises:}

1. (Standing) walk forward, raising both arms in front of your body

2. (Standing. Back facing the wall) lift your knees alternately, pulling the knee to your chest with both arms around the knee
3. (Standing. Back facing the swimming pool wall. Arms to the sides) push your legs up and forwards alternately, bending your knee backwards

4. (Standing facing the wall. Arms extended forwards touching the swimming pool edge) bend your trunk backwards to take a deep breath then bend your trunk forwards to drive out inhaled air into water

5. (Standing with hands on waist) bend your knees midway until water reaches chest level.

6. (Standing with hands on waist) take a deep breath then bend your knees completely driving out inhaled air into water (head immersed in water)

7. (Standing facing the wall. Arms thrown forward while touching the swimming pool edge) take a deep breath then bend your trunk forwards to drive out inhaled air into water.

8. (Leaning forward on both hands) bend your arms completely (inhaling outside water and exhaling under water).

9. (Standing, squatting position) Push the ground with both feet while they are pointing upwards.

10. (Leaning forward while raising both arms. Holding edge of the swimming pool) leg strokes.

11. (Sitting in the squatting position. Keeping shoulders at the water surface level. Holding $3 \mathrm{~kg}$ dumbbells) bending arms completely.

12. (Sitting in the squatting position. Keeping shoulders on the water surface level. Holding $3 \mathrm{~kg}$ dumbbells) Raising both arms forwards

13. (.Sitting in the squatting position. Keeping shoulders on the water surface level. Holding $3 \mathrm{~kg}$ dumbbells) Raising both arms to the sides

14. (Sitting in the squatting position. Keeping shoulders on the water surface level. Holding $3 \mathrm{~kg}$ dumbbells) moving both arms to the sides 
15. (Standing. Holding $3 \mathrm{~kg}$ dumbbells) walking forwards, bending both arms alternately.

16. (Standing. Holding $3 \mathrm{~kg}$ dumbbells) walking sidewards, raising both arms to the sides

\section{Cool- down:}

(Standing) raising the heels while taking a deep breath followed by a strong exhilation.

(Standing. Inclination) Moving both arms downward while regulating the respiration process.

(Standing.) Swinging forward while regulating the respiration process
(Standing) Vertical swinging while regulating the respiration process

(Standing) Angle swinging by regulating the respiration process

\section{Post- measurements:}

Post - measurement testing were taken after conducting the experiment in the same conditions as the pre- measurement and were compared for statistical treatment.

\section{Module regulation}

The participant performs the warm-up exercises which are estimated at approximately 100 heart beats per minute followed by ten exercises with one set for every exercise continuously until the required training heart rate is reached :

Targeted training heart rate= $=$ Resting heart rate +

Training intensity and volume are presented in table 1.

Table (1)

Training parameters through the program

\begin{tabular}{|c|c|c|c|}
\hline Week & Intensity & Training Heart Rate & Total time per session \\
\hline 1 & $\begin{array}{c}35 \% \\
\text { Heart Rate Reserve }\end{array}$ & 122 beats per minute & $13 \mathrm{~min}$ \\
\hline 2 & $\begin{array}{c}40 \% \\
\text { Heart Rate Reserve }\end{array}$ & 123 beats per minute & $19 \mathrm{~min}$ \\
\hline 3 & $\begin{array}{c}50 \% \\
\text { Heart Rate Reserve }\end{array}$ & 127 beats per minute & $30 \mathrm{~min}$ \\
\hline 4 & $\begin{array}{c}55 \% \\
\text { Heart Rate Reserve }\end{array}$ & 130 beats per minute & $35 \mathrm{~min}$ \\
\hline 5 & $\begin{array}{c}65 \% \\
\text { Heart Rate Reserve }\end{array}$ & 137 beats per minute & $40 \mathrm{~min}$ \\
\hline 6 & $\begin{array}{c}70 \% \\
\text { Heart Rate Reserve }\end{array}$ & 138 beats per minute & $45 \mathrm{~min}$ \\
\hline
\end{tabular}

\section{Results}

Just 3 tables should be presented, table one with total sample data (as you did), table 2 with results for both exeprimental and control group pre-program variables data and statistical significant differences, and table 3 with results for both exeprimental and control group postprogram variables data and statistical significant differences. 
Table (2)

Statistical significance of research variables for the total sample (N=20): this is a table with descriptive parameters, not statistical significance

\begin{tabular}{|c|c|c|c|c|}
\hline $\begin{array}{c}\text { Statistical } \\
\text { significanee }\end{array}$ & $\begin{array}{c}\text { Arithmetic } \\
\text { mean }\end{array}$ & $\begin{array}{c}\text { Standard } \\
\text { deviation }\end{array}$ & Kurtosis & $\begin{array}{c}\text { Coefficient of } \\
\text { variation rate }\end{array}$ \\
\hline Variables & 181.80 & 7.06 & -1.22 & 3.88 \\
\hline Diabetes & 218.65 & 19.36 & -0.75 & 8.85 \\
\hline Cholesterol & 117.80 & 2.88 & -0.79 & 2.44 \\
\hline Triglyceride & 9.30 & 4.59 & -0.74 & 5.08 \\
\hline Resting heart rate & 174.65 & 11.45 & -1.62 & 6.56 \\
\hline Exercise heart rate & 17.20 & 1.02 & 0.20 & 5.91 \\
\hline Exercise time & 152.80 & 12.08 & -1.23 & 7.91 \\
\hline Recovery heart rate- 2 minutes & 133.10 & 10.73 & -1.32 & 8.06 \\
\hline Recovery heart rate- 4 minutes & 118.20 & 8.24 & 0.25 & 6.97 \\
\hline Recovery heart rate- 6 minutes & 95.10 & 8.09 & -0.55 & 8.51 \\
\hline Body weight & & \\
\hline
\end{tabular}

Table 1 shows that all variables have normal distributionf (skewness \pm 3 ). In addition, there is evident homogeneity of the sample (CV between 2.44 and 8.85 ).

Table (3)

Differences of research variables in the experimental and the control groups before program implementation

\begin{tabular}{|c|c|c|c|c|c|c|}
\hline \multirow{2}{*}{$\begin{array}{c}\text { Statistical } \\
\text { Significance } \\
\text { Variables-Took at the table } \\
\text { above! }\end{array}$} & \multicolumn{2}{|c|}{$\begin{array}{c}\text { Experimental } \\
\text { Group } \\
\end{array}$} & \multicolumn{2}{|c|}{ Control group } & \multirow{2}{*}{$\begin{array}{c}\text { The } \\
\text { Differences } \\
\text { between the } \\
\text { two means }\end{array}$} & \multirow{2}{*}{$\begin{array}{c}\text { The } \\
\text { calculated } \\
\text { T value }\end{array}$} \\
\hline & Mean & Std \pm & Mean & Std \pm & & \\
\hline Diabetes & 181.20 & 7.33 & 182.40 & 7.12 & 1.20 & 0.371 \\
\hline Cholesterol & 219.20 & 19.75 & 218.10 & 20.00 & 1.10 & 0.124 \\
\hline Triglyceride & 117.50 & 3.14 & 118.10 & 2.73 & 0.60 & 0.457 \\
\hline Rest heart rate & 89.70 & 4.27 & 90.90 & 5.04 & 1.20 & 0.574 \\
\hline Effort heart rate & 174.20 & 12.10 & 175.10 & 11.41 & 0.90 & 0.171 \\
\hline Time for resisting effort & 17.17 & 1.10 & 17.23 & 0.98 & 0.60 & 0.129 \\
\hline Recovery after 2 minutes & 152.50 & 12.75 & 153.10 & 12.06 & 0.60 & 0.108 \\
\hline Recovery after 4 minutes & 132.70 & 11.49 & 133.50 & 10.51 & 0.80 & 0.162 \\
\hline Recovery after 6 minutes & 117.80 & 8.63 & 118.60 & 8.29 & 0.80 & 0.211 \\
\hline Body weight & 95.00 & 8.33 & 95.20 & 8.30 & 0.20 & 0.054 \\
\hline
\end{tabular}

(T) index value at the level of $0.05^{*}=2.021$

There were no statistically significant differences research variables before program implementation. 
Table (4)

Statistical significance of research variables in the control group after conducting the experiment

\begin{tabular}{|c|c|c|c|c|c|c|c|}
\hline \multirow{2}{*}{$\begin{array}{c}\text { Statistical } \\
\text { Significance }\end{array}$} & \multicolumn{2}{|c|}{$\begin{array}{c}\text { Experimental } \\
\text { group }\end{array}$} & \multicolumn{2}{c|}{ Control group } & $\begin{array}{c}\text { The } \\
\text { Difference } \\
\text { between } \\
\text { the two } \\
\text { Mariables }\end{array}$ & $\begin{array}{c}\text { Means } \\
\text { Deviation } \\
\text { of } \\
\text { Differences }\end{array}$ & $\begin{array}{c}\text { Salculated T } \\
\text { Value }\end{array}$ \\
\hline Diabetes & 149.90 & 21.81 & 186.40 & 8.29 & 36.50 & $4.95 *$ & $24.35 \%$ \\
\hline Cholesterol & 199.40 & 12.27 & 225.70 & 18.84 & 26.30 & $3.70 *$ & $13.19 \%$ \\
\hline Triglyceride & 96.40 & 7.72 & 122.90 & 2.38 & 26.50 & $9.94 *$ & $27.49 \%$ \\
\hline Rest Heart rate & 81.80 & 5.71 & 92.40 & 5.52 & 10.60 & $4.22 *$ & $12.96 \%$ \\
\hline Effort heart rate & 157.60 & 9.11 & 174.10 & 12.90 & 16.50 & $3.31 *$ & $10.47 \%$ \\
\hline Time for resisting effort & 21.93 & 3.46 & 16.41 & 1.33 & 5.52 & $4.71 *$ & $25.15 \%$ \\
\hline Recovery after 2 minutes & 136.30 & 9.31 & 160.80 & 13.24 & 24.50 & $4.79 *$ & $17.98 \%$ \\
\hline Recovery after 4 minutes & 118.60 & 7.49 & 142.50 & 12.50 & 23.90 & $5.19 *$ & $20.15 \%$ \\
\hline Recovery after 6 minutes & 98.00 & 3.46 & 118.70 & 12.11 & 20.70 & $5.20 *$ & $21.12 \%$ \\
\hline Body weight & 85.90 & 3.70 & 93.10 & 8.06 & 7.20 & $2.57 *$ & $8.38 \%$ \\
\hline
\end{tabular}

(T) index value at the level of $0.05 *=2.021$

According to Table (4), which represents Statistical significance of research variables in the experimental and the control groups after conducting the experiment, there are statistically significant differences in research variables, as the calculated $\mathrm{T}$ value was higher than the indexed $\mathrm{T}$ value at 0.05 , which means that experimental group is better than the control group in all research variables.

\section{Discussion:}

\section{1 - Diabetes}

Blood glucose level was significantly reduced in the experimental group compared to the control group after the six week exercise program. This can be attributed to the effect of applying the aerobic exercise method by using the aqueous medium,which is in conformity with the results of the studies conducted by Gleim (10:21), Shalaby Mohamed (2: 12) and MostafaNour (3 $: 115)$. They stated that the level of low fats is reduced as a result of the physical training and the increase in energy consumption in the aerobic exercises, in addition to the fact that the energy consumption rate increases during the exercise in the aqueous medium. The reduction of the blood glucose level is attributed to the physical effort exerted when using the aqueous medium which leads to higher sensitivity of body cells to the insulin hormone whose efficacy and activity increases inside the body.

\section{Cholesterol}

Experimental group showed a significant decrease in cholesterol level in comparison with the control group after the training program . . This decrease could be attributed to the specifi training program conducted. This is in conformity with the findings of Morris (15:89) who stated that cholesterol level decreases due to the decrease of fats in the body when using it as fuel for the energy during the activity after using carbohydrates as the main source of energy.

\section{Triglyceride:}

It is obvious from Table (4) that the triglyceride level decreased in the experimental group in comparison with the control group and this decrease in the triglyceride level was statistically significant. This decrease is attributed to practicing the aerobic exercises in an aqueous medium. This is in conformity with results reached by Lamp Man (14:87) and Nikkle (17:137) who stated that regular physical activity has an efficient impact in lowering the triglyceride level.

\section{Heart rate:}


From Tables (4) it can be seen that heart rate was lower in the experimental group, at rest or at maximum physical effort, in comparison with the control group, whose heart rate increased. The decreasing rate in the experimental group was statistically significant. The decrease is attributed to the improvement of the functional efficiency of the heart which is due to the fact that the program exercises include low and intensity progressive aerobic training loads, which is in accordance with Kannel's study (12:86). The physical training reduces the sympathetic activity of the heart and this provides the heart with a biological value due to the increase in blood pumped into the heart and reduction of the heart mechanism load.

\section{Physical effort endurance time per minute:}

It is obvious from Table (4) that there are statistically significant differences between the control and the experimental groups in the physical effort endurance time per minute favoring the experimental group. This is attributed to exercising of the suggested training program comprising aerobic training loads by the experimental group, which improves the efficiency of the circulatory and respiratory systems and this is in conformity with the results of studies by Consilman (7:91), Kiessling (13:14) and Ahmed El Esawi (1:13). In fact, this type of training improves the individual's efficiency and enables him to perform the required effort in a better way and for a longer time and with the same level of efficiency.

This is attributed to applying the training program comprising low-intensity training loads exercises, which improve the functional efficiency of the circulatory and respiratory systems. This improvement can also be noted in the increase of physical capacity achievement. This is in conformity with results of the studies by Morgan et al (16:87), David (17:532) who stated that the regularly performed exercises increase the mitochondria number in the skeletal muscle together with an increase in the capacity of theATP production..

Heart rate recovery after 2, 4 and 6 minutes of exertion treadmill test:
It is obvious from the Table (4) that there are statistically significant differences between the control and the experimental groups in the heart rate after 2, 4 and 6 minutes of maximum physical effort in the post-measurement, in favor of the experimental group. The decrease (improvement), in the heart rate recovery in 2, 4 and 6 minutes of maximum physical effort in the experimental group could be attributed to the exercise program comprising of lowintensity training loads. Such simulicould led to an improvement in the heart functional efficiency, thus enabling it to perform the required effort in an economic manner. Faster heart rate recovery can be attributed to improve the vagal tone and parasympathetic nervous system activity. This is in conformity with results of studies by Robinson (19:165), Obranetal (18:735) and Blomqvist(4:9) who stated that the speedy return of the heart rate to the normal state reflects the good condition of the vital organs, especially the circulatory and respiratory systems.

Table illustrate the lower rates achieved by the control group in most of the research variables. This is attributed to the fact that the control group did not participate in the proposed training program and did not participate regularly in any other training program. This is obvious from the comparison of results achieved by this group in pre-measurements and post-measurements.

\section{Body weight}

It is obvious from Table (4) the reduction in the body weight in the experimental group in comparison with the control group, whose participants put on weight. This loss of weight was may be a result of participating in the training program and burning more calories. This is in conformity with the study results, i.e., the weight loss is an indication of the oxidation of fats in the body cells under the effect of aerobic training exercises and this is also in conformity results of the study by Rvotie et al (20:760) which stated that exercises in an aqueous medium improve the physiological state of the body and have an effect on weight reduction.

\section{Conclusions:}


Performing a physical exercises program in an aqueous medium for a six week, three times a week, can improve several health-related parameters of patients suffering from degenerative diseases, compared to patients who only receive medical treatment.

\section{Recommendations}

Following recommendations can be made:

- Patients suffering from degenerative diseases could improve their health status by applying water-based exercise programs.

\section{References}

1. Ahmed Ali El Esawi: 1995, the suggested training program impact on physical fitness to improve the circulatory and respiratory system efficiency for frogmen members, unpublished master, Faculty of Physical Education for Boys at Alexandria University.

2. ShalabyMohamadShalaby: 1988, the suggested training program impact of physical exercises on some psychological and physiological variables on patients suffering from diabetes, journal and theories applications of the Faculty of Physical Education for Boys at Alexandria University, fifth issue.

3. MostafaMohamadNour: Compatibility neuromuscular exercises impact for improving the function and psychological conditions of individuals suffering of diabetes, unpublished master, Faculty of Physical Education for Boys at Alexandria University.

4. Bcomqvistyg.1995 Electrocardiogram The Frank lead exercise, acta.second.(supp). 440

5. Brav Wald: heart Disease, New York book, 1998

6. David R., Fitness and life time commitment Burgess publishing company, Minnesta

7. Knosslman, Kras Now, S. (1995) Heart Diseases and Exercises

8. Doublas: heart diseases in woman, 1988
9. FiterGiralled (1988) The American environmental movement New York

10. Glein, G.W., Nicholas J.A.,: metabolic costs and heart rate responses to treadmill walking in water at different depth and imperators, A.M.J of spots med. Vol. 1'7, No. 2, 1989.

11. Helbebr et.al: (1988) hand book of heart

12. Kannel, W.B., and Dawber, Atherosclerosis \& Pediatric Problem, Pediatr. 8.

13. Kiess Lind and Lundqvist, C.G (1999) Number and Size of Skeletal Muscle Mitochondria in Trained Sedentary Men. Mallemberg Baltimore University Park Press

14. Lamp: Physiology of Exercise response and Adaption, New York, 1992

15. Morris, H.I.,: Exercise Physiology energy nutrition and human performance, $2^{\text {nd }}$, edition, 1992

16. Morgan To, Moris H.I., (1998) Exercise Physiology Energy Nutrition and Human Performance $2^{\text {nd }}$ ed lea

17. Nikki Mcanton (1988) Effect of Physical Activity on Post Prandial leeles, Lancent, June

18. O. Brien, k.p., Lawrence (1997) Comparison During Angina Induced By Aerial Pacing. Circulation 39.

19. Robinson.T.R., (1997) Relation of Heart Rate and Systolic Blood, New York, 307

20. Rvti, R.G., Troup, J.T., Berger, R.A.,: The Effects of Non Swimming Water exercise an Older Adults, J. of Orthopedic, Sports Physical, vol. 19 No. 3, 1994

21. Smith, Wynn (1998) Cardiovascular Disease

22. Stein, Mary (1989) Cardiovascular Disease Evaluation and Prevention

23. WHO (2005) Heath Hazards of the Human Environment 